\title{
L'Assurance Italienne et l'Actuel Régime de Restrictions des Changes
}

\author{
par Alfonso Desiata *
}

1. De par sa nature, l'assurance implique de grands espaces, de vastes horizons, une ouverture totale au marché international. Afin de remplir au mieux sa tâche, l'assurance refuse toutes restrictions techniques et monétaires à son expansion.

Il existe d'ailleurs, à l'époque actuelle, des forces opposées, dont la coordination ne se vérifie que rarement en vue d'objectifs bien précis : il existe au contraire des forces d'inertie, d'accrochage, de viscosité, qui engendrent des désorientations chez tous les opérateurs, et en particulier chez les assureurs : elles altèrent - du point de vue compétitif - les conditions égales et nécessaires à l'exercice de ce secteur, en favorisant tantôt l'un tantôt l'autre concurrent.

Le secteur de l'assurance italienne constitue une sorte de laboratoire où il est plus facile de découvrir les contradictions et les asymétries concurrentielles qui se sont cumulées au fil des dernières années sous la poussée des décisions prises par de nombreux centres de pouvoir nationaux et communautaires.

Il faut tout d'abord souligner que :

1) Au cours des vingt-cinq dernières années le secteur italien de l'assurance a été aligné au point de vue technique et gestionnaire aux marchés les plus développés, suivant l'évolution technologique de notre système économique. Le marché de l'assurance est fort concurrentiel tant en ce qui concerne les conditions de couverture des risques que dans le cadre du niveau moyen des primes requises.

2) Le secteur italien de l'assurance ne craint pas la concurrence internationale, pourvu qu'il ne soit pas forcé à opérer dans des conditions d'infériorité : l'assurance italienne dont la présence internationale se manifeste tant directement qu'indirectement, par l'intermédiaire de sociétés contrôlées à l'étranger - est appréciée partout de par sa fiabilité, son niveau technique et sa solidité financière, ce qui a pu être atteint grâce à la possibilité, pour l'assureur italien à l'étranger, d'affronter ses concurrents étrangers sur un pied d'égalité.

3) Une compagnie d'assurances effectue non seulement une compensation des risques et entre risques, mais encore une redistribution des bénéfices parmi tous les assurés vie et non-vie.

* Administrateur Délégué, Generali, Trieste. 
Les assurés vie participent à la redistribution des résultats financiers des entreprises d'assurances grâce à des conditions de police données: celles-ci prévoient un taux technique de capitalisation et une participation auxdits bénéfices sous une forme « ouverte", une forme proportionnée aux résultats obtenus par des branches données ou sous une forme prédéterminée, se basant sur des mécanismes automatiques liés à la tendance de l'inflation interne ou aux oscillations de la lire italienne par rapport à l'Ecu. Les assurés non-vie jouissent, eux, d'une participation indirecte aux résultats financiers de la compagnie, et cela non pas sur la base des conditions de police, mais par le biais d'une réduction des primes payées: une pratique critiquable, mais courante. Les compagnies d'assurances opèrent en fait avec des résultats techniques fort déficitaires et les pertes techniques reflètent globalement l'insuffisance des primes payées par rapport aux règlements des sinistres effectués par la compagnie et aux frais soutenus par celle-ci. Les résultats techniques négatifs sont compensés par les résultats financiers obtenus à travers l'investissement des actifs à couverture des réserves techniques.

2. Il va de soi que d'autant plus consistants sont les résultats financiers provenant des placements d'assurance, d'autant plus élevée sera la participation aux bénéfices prédéterminée en faveur des assurés. Et encore : les résultats financiers obtenus au moyen des placements sont directement proportionnés aux niveaux des taux réels que l'on peut réaliser sur le marché financier.

Si le marché italien de l'assurance était un marché «fermé », sans contacts directs ou indirects avec l'étranger, la couverture des risques ne serait assurée que par les Compagnies opérant en Italie. Le libre jeu de la concurrence se ferait correctement, toute compagnie étant soumise aux mêmes obligations prévues par notre système en ce qui concerne l'exploitation et toutes étant également tenues d'opérer sur le même marché des capitaux en ce qui concerne la fonction financière. Au contraire - et ce ne pourrait pas être autrement - notre marché de l'assurance est ouvert à la composante internationale et la présence de la concurrence étrangère entraîne une rupture de l'égalité des conditions de celle-ci, et cela non pas du point de vue technique ou gestionnaire, mais du côté financier.

L'assureur étranger, qui - soit directement, soit au moyen de la réassurance, de la co-assurance ou bien en recourant à d'autres systèmes - couvre un risque italien en monnaie nationale, transfère à l'étranger le service des provisions techniques, de la réserve pour primes non acquises et de la provision pour sinistres à payer, en employant sur d'autres marchés sa contrepartie financière dans l'espoir d'obtenir un taux d'intérêt réel plus élevé que celui qu'il réaliserait en Italie. Mais l'assureur italien n'a pas cette liberté, à cause des restrictions de change auxquelles il est soumis : c'est ainsi qu'il se trouve à devoir opérer dans des conditions d'infériorité du côté financier. Le contrat d'assurance étant un tout unique, ce désavantage se répercute sur tous les autres aspects dudit contrat, en excluant du marché l'assureur national.

Et cela se vérifie dans la pratique de tous les jours, puisqu'en Italie le taux d'intérêt réel est - depuis des années - constamment inférieur aux taux pratiqués sur les plus importants marchés financiers étrangers. En nous limitant à l'année 1982, sur la base des données OCDE, nous pouvons fournir les indications suivantes: 


\begin{tabular}{lccc} 
& $\begin{array}{c}\text { Prime rate } \\
\%\end{array}$ & $\begin{array}{c}\text { Inflation } 1982 \\
\%\end{array}$ & $\begin{array}{c}\text { Ecart } \\
\%\end{array}$ \\
\cline { 2 - 4 } Italie & 19,50 & 16,40 & 3,10 \\
Etats-Unis & 10,50 & 3,90 & 6,60 \\
Japon & 6,00 & 1,80 & 4,20 \\
RFA & 8,00 & 4,60 & 3,40 \\
France & 12,25 & 9,70 & 2,55 \\
Royaume-Uni & 10,50 & 5,40 & 5,10
\end{tabular}

En conclusion, la participation aux bénéfices des assurés fait ressortir la nécessité d'une égalité de conditions de la fonction financière existant parmi toutes les compagnies d'assurances concurrentes, tant nationales qu'internationales, à moins que nous ne voulions assister à une lente mais inévitable décadence des compagnies italiennes.

3. Voyons donc quelques détails opérationnels afin de donner une image aussi transparente que possible de l'asymétrie concurrentielle existant sur le marché italien au détriment des compagnies d'assurance nationales et de découvrir quelques solutions, certes pas de nature conservatrice, mais visant à obtenir une majeure liberté d'emploi pour notre secteur.

Si la fonction financière est le facteur discriminant, la liberté d'établissement soutenue par la Communauté Economique Européenne ne crée aucun problème qui ne puisse être résolu. C'est plutôt la libre prestation des services qui soulève des complications: la proposition de directive adoptée en 1975 est encore à l'examen des institutions communautaires, de même que la co-assurance qui aurait dû entrer en vigueur au mois de juin 1980.

Exception faite pour beaucoup d'autres aspects de nature institutionnelle et de contrôle technique, la libre prestation des services impliquerait nécessairement une discrimination dans le domaine de la fonction financière au détriment total des compagnies nationales obligées à opérer exclusivement sur le marché financier italien. Une coexistence entre opérateurs qui soient discriminés sous le profil de la fonction financière se révèle difficile : d'un côté, on se trouverait face à une foule d'assureurs totalement libres de choisir le marché et la devise susceptibles de maximiser la fonction de rentabilité des placements, et de l'autre une foule d'assureurs obligés à opérer à l'intérieur de la camisole de force des restrictions des changes, de sorte que l'écart du taux d'intérêt réel défavoriserait l'assureur italien. La même chose s'avère pour la co-assurance communautaire, qui est pourtant limitée au secteur des grands risques industriels et commerciaux qui dépassent certaines limites fixées.

La solution ne peut être que graduelle. Avant tout, la libre prestation des services et la co-assurance communautaire pourraient être limitées au secteur des risques industriels et commerciaux d'une certaine importance. La prise en charge du risque et le contrat y afférent devraient être exprimés en Ecus et les assureurs nationaux et communautaires seraient ainsi libres de placer la contre-valeur en Ecus dans n'importe quel marché communautaire dans la même proportion des monnaies nationales dont se compose l'unité 
de compte européenne. Même si cela n'entraînait pas l'élimination totale de la distorsion de la fonction financière, on pourrait la réduire considérablement. De plus, on éviterait l'altération des déséquilibres des marchés nationaux d'assurance en limitant aux gros risques l'activité liée à la libre prestation des services et à la co-assurance communautaire : on opérerait ainsi correctement dans le domaine de certains risques vis-à-vis desquels les risk-managers utilisent depuis longtemps les monnaies «fortes" plutôt que la lire italienne. On pourrait ainsi élargir le domaine des contrats libellés en monnaie étrangère - bien que dans une devise particulière - à d'autres risques par rapport à la situation existant en la matière, limitée à des risques donnés tels que les corps des bateaux. Une fois cette phase expérimentale terminée et un certain bagage de connaissances acquis, on pourrait procéder aux amendements nécessaires. Aussi, sur la base de cette expérience, pourrait-on créer un marché européen de titres exprimés en Ecus, un système qui s'était largement répandu en 1982, année qui fut ainsi dénommée «l'année Ecu ».

4. La subordination à la concurrence étrangère de l'activité d'assurance sur notre marché ne dépend pas seulement de la discrimination monétaire, mais encore d'autres éléments, comme par exemple la composante fiscale.

De récentes dispositions, prévoyant des alourdissements des charges fiscales sur l'assurance, favorisent ultérieurement la concurrence étrangère au détriment de la concurrence nationale, en faisant affluer certains secteurs de l'activité nationale sur d'autres marchés de l'assurance et de la co-assurance. Cela entraîne un appauvrissement marqué de notre marché et en réduit la capacité de rétention, en augmentant ainsi le résultat déficitaire de notre balance des paiements en matière de services d'assurance. $\mathrm{A}$ ce propos, il faut rappeler que ce déficit est, depuis des années, de l'ordre d'environ cent milliards de lires par an.

5. D'autres problèmes se manifestent dans le domaine des nouveaux produits vie, tels que l'assurance mixte ou l'assurance du capital différé, dont les coûts d'acquisition sont très élevés et dépassent largement la prime de la première année.

C'est ainsi que la compagnie d'assurance, qu'elle soit grande ou petite, atteindra bientôt ses limites d'auto-financement, d'où la nécessité de s'orienter - en ce qui concerne les financements - vers la réassurance, afin de faire face au rythme de développement de la branche vie : l'entreprise cherchera à placer la réassurance sur le marché local de sorte à alléger ses problèmes de financement.

Il peut quand même arriver - comme c'est aujourd'hui le cas de l'Italie - qu'un développement exceptionnel de la production vie ne soit pas un fait spécifique se vérifiant auprès d'une seule compagnie, mais une circonstance commune à tout le marché : il est évident que dans une telle situation il sera difficile de trouver sur place un réassureur pouvant prendre en charge la production de la concurrence, d'où la nécessité de recourir au marché international de l'assurance, qui examinera cette opération sous l'aspect purement financier. C'est à ce moment qu'il faut tenir compte de l'incertitude de la situation économique italienne et de la fragilité de la lire sur le marché international des changes.

Un contrat de réassurance implique en fait pour le réassureur le paiement de sa quote-part de financement qui sera récupérée, au cours de la durée de chaque police, à travers l'encaissement des soldes annuels auprès de la cédante. Cependant ces soldes seront 
payés en lires italiennes, de sorte que le réassureur étranger - très probablement - ne pourra récupérer le financement affectué en devises à cause de la dévaluation prévisible que la lire subira vis-à-vis des monnaies «fortes". Même si cela ne se vérifie pas, le réassureur étranger, outre le risque d'assurance, devra couvrir aussi le risque monétaire. De plus, les réserves mathématiques (y inclus la quote-part réassurée) doivent rester « sub lege " en dépôt auprès de la cédante, de sorte que le réassureur ne peut pas les utiliser.

D'où l'opportunite d'éliminer - au moins partiellement - le risque monétaire que le réassureur international n'accepte pas très volontiers. On pourrait atteindre cet objectif:

a) en consentant aux réassureurs étrangers de couvrir leurs réserves moyennant des titres étrangers en dépôt auprès de la cédante ;

b) en consentant aux réassureurs étrangers de placer leurs réserves hors d'Italie et de donner aux cédantes de garanties consistantes par l'intermédiaire de lettres de crédit ad hoc.

6. Nous avons parlé au début de la présence massive de l'assurance italienne dans les marchés internationaux, soit sous une forme directe soit indirectement par l'intermédiaire des compagnies contrôlées par des groupes nationaux.

Dans les deux cas, les points discriminants sous l'aspect de la fonction monétaire sont considérés comme insignifiants par les techniciens : lorsqu'il s'agit des succursales, celles-ci opèrent évidemment sur un pied d'égalité vis-à-vis de la concurrence étrangère des autrès pays; lorsqu'il s'agit d'une activité directe, réalisée par l'intermédiaire des directions, représentations et agences, cela n'entraîne aucune restriction monétaire particulièrement sévère de la part des autorités italiennes. Et notre expérience nous confirme que la présence de l'assurance italienne à l'étranger s'est renforcée en obtenant progressivement du respect et du prestige.

En revanche, l'activité italienne de réassurance à l'étranger se présente sous un aspect différent : elle est soumise à certaines restrictions quant à l'exploitation des opérations monétaires fixées par la réglementation italienne des changes. Dans une phase historique caractérisée par la rapidité des communications et par l'extrême agilité des marchés monétaires et financiers, les dispositions italiennes en la matière nécessitent une révision rigoureuse en ce qui concerne : les portefeuilles des compagnies d'assurance ; la possibilité d'accepter, entre certaines limites, que l'exploitation puisse se dérouler même sur des marchés à terme ; la suppression des limitations opérationnelles sur les titres bancaires et sur les titres appartenant à des holdings financières, pourvu que celles-ci soient cotées ; reconnaître que le marché monétaire ne consiste pas seulement dans des dépôts bancaires, mais qu'il comprend beaucoup d'autres titres de crédit, tels que les certificats de dépôts et les acceptations bancaires ; reconnaître définitivement aux lettres de crédit et à d'autres titres similaires le droit d'être utilisés; admettre finalement que l'exploitation des obligations d'un portefeuille puisse viser même des titres non cotés.

En conclusion, il faut souligner le fait que les assureurs italiens ne demandent qu'un peu de bon sens et le sens pratique de la réalité opérationnelle de nos jours : ils n'exigent aucun privilège, mais tout simplement la parité concurrentielle des conditions de départ: bref, que l'internationalisme italien ne soit pas purement nominal, mais une réalité effective, riche en décisions importantes et, surtout, coordonnées. 